\title{
BMJ Open Using an integrated knowledge translation or other research partnership approach in trainee-led research: a scoping review protocol
}

\author{
Christine E Cassidy (10 , ${ }^{1}$ Amy Jane Beck, ${ }^{2}$ Aislinn Conway (D) , ${ }^{3}$ \\ Melissa Demery Varin, ${ }^{4}$ Celia Laur, ${ }^{5}$ Krystina B Lewis, ${ }^{6,7}$ Emily R Ramage (D) ,8 \\ Tram Nguyen, ${ }^{9,10}$ Sandy Steinwender, ${ }^{11}$ Ilja Ormel, ${ }^{12}$ Lillian Stratton, ${ }^{1}$ \\ Hwayeon Danielle Shin ${ }^{1}$
}

To cite: Cassidy CE, Beck AJ, Conway $\mathrm{A}$, et al. Using an integrated knowledge translation or other research partnership approach in trainee-led research: a scoping review protocol. BMJ Open 2021;11:e043756. doi:10.1136/ bmjopen-2020-043756

- Prepublication history and additional supplemental material for this paper are available online. To view these files, please visit the journal online (http://dx.doi.org/10.1136/ bmjopen-2020-043756).

Received 12 August 2020 Accepted 07 May 2021

Check for updates

(C) Author(s) (or their employer(s)) 2021. Re-use permitted under CC BY-NC. No commercial re-use. See rights and permissions. Published by BMJ.

For numbered affiliations see end of article.

Correspondence to Dr Christine E Cassidy; ccassidy@dal.ca

\section{ABSTRACT}

Introduction Collaborative research approaches, such as co-production, co-design, engaged scholarship and integrated knowledge translation (IKT), aim to bridge the evidence to practice and policy gap. There are multiple benefits of collaborative research approaches, but studies report many challenges with establishing and maintaining research partnerships. Researchers often do not have the opportunity to learn how to build collaborative relationships, and most graduate students do not receive formal training in research partnerships. We are unlikely to make meaningful progress in strengthening graduate and postgraduate training on working collaboratively with the health system until we have a better understanding of how students are currently engaging in research partnership approaches. In response, this scoping review aims to map and characterise the evidence related to using an IKT or other research partnership approach from the perspective of health research trainees.

Methods and analysis We will employ methods described by the Joanna Briggs Institute and Arksey and 0'Malley's framework for conducting scoping reviews. The reporting will follow the Preferred Reporting Items for Systematic Reviews and MetaAnalysis extension for scoping reviews checklist. We will include both published and unpublished grey literature and search the following databases: MEDLINE, Embase, CINAHL, PsycINF0, ProQuest Dissertations \& Theses Global databases, Google Scholar and websites from professional bodies and other organisations. Two reviewers will independently screen the articles and extract data using a standardised data collection form. We will narratively describe quantitative data and conduct a thematic analysis of qualitative data. We will map the IKT and other research partnership activities onto the Knowledge to Action cycle and IAP2 Levels of Engagement Framework.

Ethics and dissemination No ethical approval is required for this study. We will share the results in a peer-reviewed, open access publication, conference presentation and stakeholder communications.
Strengths and limitations of this study

- We will follow established scoping review methods described by the Joanna Briggs Institute and Arksey and 0'Malley and report the review using the Preferred Reporting Items for Systematic Reviews and Meta-Analysis extension for scoping reviews checklist.

- The study selection, data extraction and charting will be performed by two independent reviewers to minimise the risk of bias or errors.

- Although comprehensive, this scoping review has limitations regarding the number of databases and English language, and may limit research from lowincome and middle-income countries.

- There are many different types of health research partnership approaches. Despite including a range of search terms, our strategy may not retrieve all papers related to health research partnerships.

- Although we will conduct a follow-up search of the author's information to identify trainee status, it is possible that we may not capture all trainees who have used an integrated knowledge translation or other research partnership approach in their work.

\section{INTRODUCTION}

Challenges persist in implementing and sustaining the use of evidence in healthcare practice and policy. Recent evidence suggests that on average only $60 \%$ of healthcare is consistent with evidence-based guidelines, $30 \%$ of care practices have low therapeutic value and $10 \%$ of care is harmful. ${ }^{1}$ Collaborative research approaches, such as co-production, co-design, engaged scholarship and integrated knowledge translation (IKT), ${ }^{2}$ aim to bridge the evidence to practice and policy gap by producing relevant research to address health system problems. IKT, specifically, focuses on bridging the gap through research partnerships. IKT is defined as 'a 
model of collaborative research, where researchers work with knowledge users who identify a problem and have the authority to implement the research recommendations' (Kothari et al, ${ }^{3}$ p299). Benefits of IKT and other research partnership approaches include improved quality of research, ${ }^{4}$ enhanced value for research among decision makers, ${ }^{5}$ increased capacity among decision makers for engaging in research ${ }^{5-7}$ and more impactful and useful research findings. ${ }^{489}$

There are increasing expectations for researchers and knowledge users in the health system (ie, patients, families, care partner, clinicians, decision makers, policy makers and funders) to use a research partnership approach, such as IKT, to increase the relevance and use of research findings in health practice, programmes and policies. ${ }^{3}{ }^{10}$ However, collaborative research is not without challenges; studies report many challenges with establishing and maintaining research partnerships with knowledge users. ${ }^{4511}$ Significant time is needed to develop trusting, authentic relationships; and there may be insufficient resources to support partnership development and maintenance. ${ }^{11}$ Furthermore, differing needs and priorities among researchers and health system decision makers, ${ }^{4511}$ as well as unclear goals, roles and expectations, can hinder research partnerships. ${ }^{5}$

A lack of skill or understanding of the collaboration process has also been identified as an important barrier to effective research partnerships with knowledge users. ${ }^{5}$ Studies have shown that effectiveness in collaborative health research requires researchers to have specific knowledge and skills for working in partnership with health system decision makers. ${ }^{12}$ Researchers often lack an understanding of the health system context and skills to engage in collaborative work. ${ }^{13}$ This can lead to ineffective researcher behaviour and affect the development of positive, mutually beneficial research partnerships. ${ }^{13}$

Researchers often do not have the opportunity to learn how to establish effective collaborative relationships with knowledge users in the health system. ${ }^{11}$ Most graduate students do not receive formal training in collaborative health research approaches. ${ }^{14}{ }^{15}$ Graduate students and postdoctoral trainees who engage in research partnerships with knowledge users are often self-motivated and supported with experiential learning opportunities or are supervised or receive mentorship from established researchers with expertise in IKT or other research partnership approaches. ${ }^{16}$ However, it is imperative that all health research trainees learn how to build trusting, effective relationships with knowledge users to foster meaningful, ethical research with relevant outcomes. ${ }^{17}$ A survey of PhD-prepared research highlighted unmet learning needs related to collaboration in research during their training. ${ }^{18}$ As such, efforts are needed to improve academic preparation for engaging in health research partnerships. ${ }^{1316}$

Given the challenges in using an IKT and other research partnership approaches, efforts are needed to support health research graduate trainees in engaging in research partnerships with knowledge users. Currently, the research partnership literature illustrates the benefits and challenges to collaborative health research from the perspective of researchers and knowledge users. ${ }^{11} 13$ 19-21 However, little is known about how trainees engage in research partnership approaches, such as IKT. Therefore, the purpose of this scoping review is to map and characterise the evidence related to using an IKT approach or other research partnerships from the perspective of health research trainees in thesis and/or postdoctoral work.

\section{METHODS}

We will conduct this scoping review using the Joanna Briggs Institute (JBI) scoping review methodology ${ }^{22}$ and Arksey and O'Malley framework for conducting scoping reviews, ${ }^{23}$ as outlined in the following five steps. We will use the Preferred Reporting Items for Systematic Reviews and Meta-Analysis (PRISMA) extension for scoping reviews ${ }^{24}$ to guide the reporting of our scoping review.

\section{Stage 1: identifying the research question}

We aim to conduct a scoping review to map and characterise the available evidence related to using an IKT or other research partnership approach from the perspectives of trainees in thesis and/or postdoctoral work via the following research question:

1. How has IKT and other research partnership approaches been applied in thesis and/or postdoctoral health research?

Additional research objectives include:

A. Identify IKT/research partnership principles, strategies and/or tools used in trainee-led health research.

B. Identify barriers and facilitators to, and recommendations for using IKT or other research partnership approaches in trainee-led health research.

C. Identify if/how outcomes were reported and evaluated in trainee-led health research using IKT or other research partnerships.

\section{Stage 2: identifying relevant studies}

To identify relevant studies, we will include key inclusion criteria based on the population, concept and context mnemonic recommended by JBI.

\section{Participants}

This review will consider literature for which health research trainees (ie, graduate students (masters, doctoral) and postdoctoral fellows) are the primary author/researcher of the article. Postdoctoral fellows may also be described as postdoctoral researchers or postdoctoral research associates. Students/fellows must be in the position of a trainee, meaning that the included studies must relate to the student or fellow's thesis/programme project/dissertation/fellowship projects.

\section{Concept}

This review will consider studies that explore IKT and other research partnership approaches in trainee-led 
Table 1 Operational terms and definitions

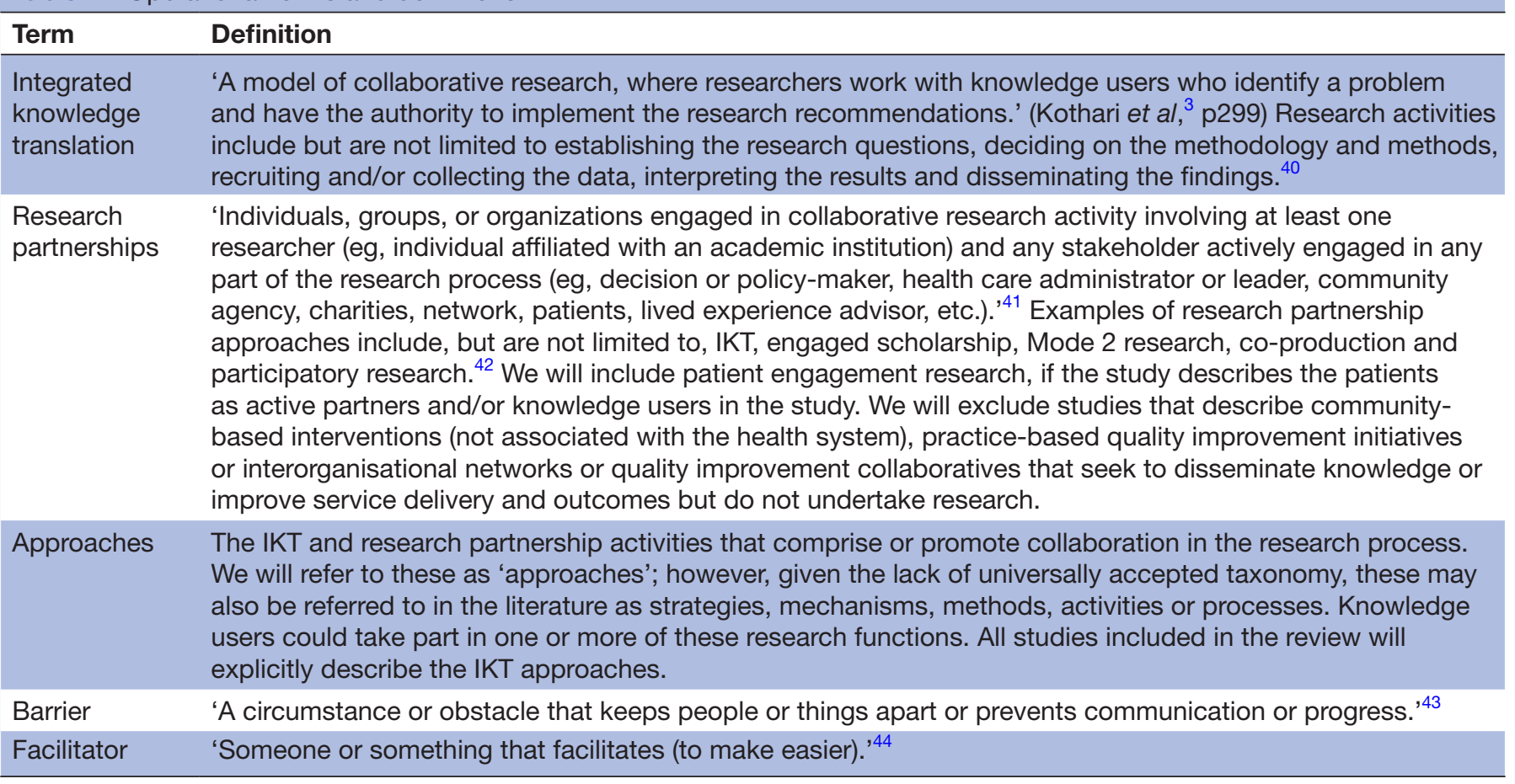

health research. The review will include only studies that describe the trainee's experience with IKT research and related partnership approaches (ie, manuscript or thesis/ dissertation chapter that provides a reflection or description of the approach, text or opinion paper describing how the partnership approach was used). Papers must explain how the research partnership approach was used, including principles, strategies and/or tools. Studies that state the use of a research partnership approach but do not describe how it was used will be excluded. Studies that describe barriers and facilitators to using an IKT or other research partnership approach will be included. Studies that evaluate the IKT approach will also be included. For the purpose of this review, we will use the following operational terms and definitions (table 1).

\section{Context}

This scoping review will consider literature focused on trainee-led health research. For the purpose of this review, health research refers to research that aims to 'increase our knowledge of health, disease, and health services, and to then apply that knowledge to help people lead healthier lives' (Government of Canada CIHR, ${ }^{25}$ p5). It may include 'biomedical research, epidemiological studies, and health services research, as well as studies of behavioural, social, and economic factors that affect health' (Gostin et al, ${ }^{26}$ para4).

\section{Search strategy}

The search strategy will be developed with a health science librarian and aims to locate both published and unpublished literature. Hoekstra and colleagues ${ }^{27}$ developed a comprehensive search strategy for synthesising the research partnership literature; we will use these search strategy findings to inform our search strategy for this scoping review. The proposed scoping review will follow the three-step process in accordance with the JBI Scoping Review Methodology. ${ }^{28}$ The search strategy aims to locate both published and unpublished primary studies, reviews and text and opinion papers. We conducted an initial limited search of MEDLINE and CINAHL to identify articles on the topic. Next, we used the text words contained in the titles and abstracts of relevant articles, and the index terms used to describe the articles to develop a full search strategy. The search strategy, including all identified keywords and index terms, will be adapted for each included information source.

We will search the following databases: MEDLINE, Embase, CINAHL and PsycINFO. The final search strategy for MEDLINE can be found in online supplemental appendix 1. We will also search sources of unpublished studies and grey literature including ProQuest Dissertations \& Theses Global databases and the first 50 pages of Google Scholar. Only studies published in English will be included. No date limits will be applied to allow for exploration of the use of IKT and/or other research partnership approaches in trainee-led research over time. We will search for grey literature resources including those identified in the Canadian Agency for Drugs and Technologies in Health grey literature checklist Grey Matters: a practical tool for searching health-related grey literature ${ }^{29} \mathrm{We}$ will use websites of research and academic institutions and health system organisation, together with concept papers, reports and blog posts. We will search relevant websites of professional bodies or organisations such as 
the Integrated Knowledge Translation Research Network (IKTRN), SPOR Units, Dissemination \& Implementation, National Institute for Health Research Collaborations for Leadership in Applied Health Research and Care and KT Canada. If appropriate, we will contact individuals or groups to source additional material (ie, Twitter, IKTRN members, relevant KT listservs). We will use our professional networks in the area of IKT and collaborative health research to email relevant content experts to identify additional sources that meet the inclusion criteria. Reference chaining will also be conducted with all included articles.

\section{Stage 3: study selection}

Following the search, we will collate all identified records, upload them to Covidence ${ }^{30}$ and remove duplicates. Two independent reviewers will screen the titles and abstracts against the inclusion and exclusion criteria for the review. Potentially relevant papers/ theses will be retrieved in full and uploaded into the Covidence software. Next, two independent reviewers will assess the full text of selected citations in detail against the inclusion criteria. We will record and report reasons for exclusion of full text papers. Any disagreements that arise between the reviewers at each stage of the selection process will be resolved through discussion, or with a third reviewer.

We will conduct a follow-up of each primary author during the full-text assessment stage for more information about their trainee status. When papers do not mention a primary author's status, we will search the author's name and the indicated affiliation in Google. If the primary author is found to be a trainee, we will include the corresponding paper. Furthermore, we will make use of available LinkedIn profiles of the authors to identify their trainee status. When necessary, we will contact that authors to clarify their trainee status. We will report the results of the search in full in the final scoping review and present them in an adapted PRISMA flow diagram. ${ }^{24}$

\section{Public and patient involvement}

There will be no public and patient engagement in this review of trainee-led research; however, as mentioned, we will consult with experts in this topic area to identify relevant sources.

\section{Stage 4: charting the data}

Two independent reviewers will use a data charting tool developed by the research team to extract data from papers included in the scoping review. A draft data charting tool is provided (see online supplementary appendix II). We will extract the following study information: author(s); year of publication; country of origin; study aim/purpose; study population; study setting; design; length of study; trainee characteristics (health/clinical discipline, affiliations with clinical institutions, stage of training); knowledge users' characteristics (level of involvement, stage of involvement, coauthors, incentives or compensation for knowledge users); description of IKT approach; IKT principles/strategies; stage of research process; barriers and facilitators; impact/outcome; and implications/ recommendations. We will define impact as 'identifiable benefit to, or positive influence on, the economy, society, public services, health, the environment, quality of life, or academia's1 and outcomes as 'a planned, a priori assessment described in the study methods that is used to determine a change in status as a result of interventions, can be measured or assessed as a component of the study, and is not something of futuristic benefit. ${ }^{32}$

We will pilot the draft data charting tool with five studies and modify as needed. Modifications will be detailed in the full scoping review. We will resolve any disagreements that arise between the reviewers through discussion with a third reviewer. Authors will be contacted to request missing or additional data, where required. Two attempts to contact authors will be made. We will wait 2 weeks before a follow-up email.

We will use several frameworks to analyse the extracted data. First, we will map included papers onto the seven phases of the Knowledge to Action cycle ${ }^{33}$ based on the reported research purpose and objectives. Second, we will categorise the knowledge users' engagement onto the five levels of public participation of the IAP2 spectrum. ${ }^{34}$ Third, we will identify the research stages that knowledge users are engaged in, including (1) development of research question, (2) development of research proposal, (3) administrative prelaunch, (4) recruitment and data collection, (5) data analysis and (6) dissemination and implementation. ${ }^{35}$ Fourth, we will use the Workgroup for Intervention Development and Evaluation Research ${ }^{36}$ reporting checklist to describe details about the IKT approach, including (1) content (nature and goal of the study and/or IKT partnership); (2) mode of delivery (specific types of IKT activities in which knowledge users were involved); (3) duration and/or frequency (timing of IKT activities); (4) participants (who were involved in specific IKT activities) and (5) personnel (who coordinated or led IKT activities) (scoping review objective A). Fifth, we will use the Capability, Opportunity and Motivation-Behaviour (COM-B) model ${ }^{37}$ and Theoretical Domains Framework (TDF) ${ }^{38}$ to characterise trainee-reported barriers and facilitators to knowledge user engagement (scoping review objective B). Both COM-B and TDF are behaviour frameworks that capture both internal and external influences on behaviour change and have been used in a range of healthcare disciplines to analyse behaviours. We will map narrative descriptions of reported barriers and facilitators onto the most appropriate domains of COM-B and TDF. Lastly, we will categorise the reported outcomes and impact of engagement to levels of patients, provider and system (scoping review objective $\mathrm{C}$ ). As data coding is expected to be an iterative process, necessary changes 
to the coding scheme will be made and reported in the full review.

\section{Stage 5: collating, summarising and reporting the results}

We will present the charted data in a tabular form that aligns with the study's objectives. In addition to the tables, we will create a figure of the barriers, facilitators, strategies, impact/outcomes and recommendations in included studies. ${ }^{28}$ We will produce descriptive numerical summaries of the quantitative data (ie, frequency of barriers and facilitators, outcomes, etc). We will conduct an inductive thematic analysis on the qualitative data. ${ }^{39}$ Lastly, a narrative summary will accompany these presentations and describe how the findings relate to the review's objective and subquestions.

\section{ETHICS AND DISSEMINATION}

Since the scoping review methodology aims at synthesising information from publicly available publications, this study does not require ethical approval. The goal of this scoping review is to map and characterise the evidence related to using an IKT approach or other research partnerships as a trainee in thesis and/or postdoctoral work. We anticipate the results of the scoping review will inform future research directions on exploring the trainee's experience with using an IKT and other research partnership approach. As such, we will share the results in a peer-reviewed, open access publication and relevant conference presentations. Furthermore, we expect the findings to help inform future training modernisation efforts. We will share the findings with key academic and health system training stakeholders through brief evidence summaries, informal presentations and targeted meetings.

\author{
Author affiliations \\ ${ }^{1}$ School of Nursing, Dalhousie University, Halifax, Nova Scotia, Canada \\ ${ }^{2}$ Faculty of Nursing, University of Calgary, Calgary, Alberta, Canada \\ ${ }^{3}$ BORN Ontario, CHEO, Ottawa, Ontario, Canada \\ ${ }^{4}$ School of Nursing, University of Ottawa Faculty of Health Sciences, Ottawa, \\ Ontario, Canada \\ ${ }^{5}$ Institute for Health System Solutions and Virtual Care, Women's College Hospital, \\ Toronto, Ontario, Canada \\ ${ }^{6}$ School of Nursing, University of Ottawa, Ottawa, Ontario, Canada \\ ${ }^{7}$ University of Ottawa Heart Institute, University of Ottawa, Ottawa, Ontario, Canada \\ ${ }^{8}$ School of Health Sciences, University of Newcastle, Callaghan, New South Wales, \\ Australia \\ ${ }^{9}$ Centre for Implementation Research, Ottawa Hospital Research Institute, Ottawa, \\ Ontario, Canada \\ ${ }^{10}$ School of Epidemiology \& Public Health, University of Ottawa, Ottawa, Ontario, \\ Canada \\ ${ }^{11}$ Faculty of Health Sciences, Western University, London, Ontario, Canada \\ ${ }^{12}$ Faculty of Medicine, McGill University, Montreal, Quebec, Canada
}

Twitter Amy Jane Beck @amyjanebeck, Aislinn Conway @AislinnConway and Emily R Ramage @EmilyRRamage

Acknowledgements We wish to thank the Integrated Knowledge Translation Research Network for their support of this study. We would also like to thank Dr. lan D Graham for reviewing an earlier draft of this protocol.

Contributors CEC, AJB, AC, MDV, CL, KBL, ERR, TN and SS designed the scoping review protocol including data collection and interpretation planning. CEC, AJB, AC,
MD-V, CL, KBL, ERR, TN, SS, I0, LS and HDS drafted and revised the protocol for intellectual content and made final approval for the submission of the protocol.

Funding The authors have not declared a specific grant for this research from any funding agency in the public, commercial or not-for-profit sectors.

Competing interests None declared.

Patient consent for publication Not required.

Provenance and peer review Not commissioned; externally peer reviewed.

Supplemental material This content has been supplied by the author(s). It has not been vetted by BMJ Publishing Group Limited (BMJ) and may not have been peer-reviewed. Any opinions or recommendations discussed are solely those of the author(s) and are not endorsed by BMJ. BMJ disclaims all liability and responsibility arising from any reliance placed on the content. Where the content includes any translated material, BMJ does not warrant the accuracy and reliability of the translations (including but not limited to local regulations, clinical guidelines, terminology, drug names and drug dosages), and is not responsible for any error and/or omissions arising from translation and adaptation or otherwise.

Open access This is an open access article distributed in accordance with the Creative Commons Attribution Non Commercial (CC BY-NC 4.0) license, which permits others to distribute, remix, adapt, build upon this work non-commercially, and license their derivative works on different terms, provided the original work is properly cited, appropriate credit is given, any changes made indicated, and the use is non-commercial. See: http://creativecommons.org/licenses/by-nc/4.0/.

\section{ORCID iDs}

Christine E Cassidy http://orcid.org/0000-0001-7770-5058

Aislinn Conway http://orcid.org/0000-0002-7566-3138

Emily R Ramage http://orcid.org/0000-0002-0599-7028

\section{REFERENCES}

1 Braithwaite J, Glasziou P, Westbrook J. The three numbers you need to know about healthcare: the 60-30-10 challenge. BMC Med 2020;18:102.

2 Rycroft-Malone J, Burton CR, Bucknall T, et al. Collaboration and Co-Production of knowledge in healthcare: opportunities and challenges. Int J Health Policy Manag 2016;5:221-3.

3 Kothari A, McCutcheon C, Graham ID. Defining integrated knowledge translation and moving forward: a response to recent commentaries. Int J Health Policy Manag 2017;6:299-300.

4 Oliver K, Kothari A, Mays N. The dark side of coproduction: do the costs outweigh the benefits for health research? Health Res Policy Syst 2019;17:33.

5 Gagliardi AR, Berta W, Kothari A, et al. Integrated knowledge translation (IKT) in health care: a scoping review. Implement Sci 2016;11:38.

6 Jagosh J, Macaulay AC, Pluye P, et al. Uncovering the benefits of participatory research: implications of a realist review for health research and practice. Milbank Q 2012;90:311-46.

7 Jessani NS, Valmeekanathan A, Babcock C, et al. Exploring the evolution of engagement between academic public health researchers and decision-makers: from initiation to dissolution. Health Res Policy Syst 2020;18:15.

8 Walter I, Davies H, Nutley S. Increasing research impact through partnerships: evidence from outside health care. J Health Serv Res Policy 2003;8(Suppl 2):58-61.

9 Hofmeyer A, Scott C, Lagendyk L. Researcher-decision-maker partnerships in health services research: practical challenges, guiding principles. BMC Health Serv Res 2012;12:280.

10 IKT Research Network. Doing research with the people who use it. Available: https://iktrn.ohri.ca/ [Accessed 12 May 2020].

11 Nyström ME, Karltun J, Keller C, et al. Collaborative and partnership research for improvement of health and social services: researcher's experiences from 20 projects. Health Res Policy Syst 2018;16:46.

12 Barratt $H$, Shaw J, Simpson L, et al. Health services research: building capacity to meet the needs of the health care system. $J$ Health Serv Res Policy 2017;22:243-9.

13 Bowen S, Botting I, Graham ID, et al. Experience of Health Leadership in Partnering with University-Based Researchers in Canada - A Call to "Re-imagine" Research. Int J Health Policy Manag 2019;8:684-99.

14 Bornstein S, Heritage M, Chudak A, et al. Development of enriched core competencies for health services and policy research. Health Serv Res 2018;53(Suppl 2):4004-23. 
15 McMahon M, Bornstein S, Brown A, et al. Training for impact: PhD modernization as a key resource for learning health systems. Healthc Policy 2019;15:10-15.

16 Cassidy CE, Bowen S, Fontaine G, et al. How to work collaboratively within the health system: workshop summary and facilitator reflection. Int J Health Policy Manag 2020;9:233-239.

17 Antes AL, Kuykendall A, DuBois JM. Leading for research excellence and integrity: a qualitative investigation of the relationship-building practices of exemplary principal Investigators. Account Res 2019;26:198-226.

18 Kyvik S, Olsen TB. The relevance of doctoral training in different labour markets. J Education Work 2012;25:205-24.

19 Zych MM, Berta WB, Gagliardi AR. Conceptualising the initiation of researcher and research user partnerships: a meta-narrative review. Health Res Policy Syst 2020;18:24.

20 Zych MM, Berta WB, Gagliardi AR. Initiation is recognized as a fundamental early phase of integrated knowledge translation (IKT): qualitative interviews with researchers and research users in IKT partnerships. BMC Health Serv Res 2019;19:772.

21 Sibbald SL, Kang H, Graham ID. Collaborative health research partnerships: a survey of researcher and knowledge-user attitudes and perceptions. Health Res Policy Syst 2019;17:92.

22 Peters M, Godfrey C, Mclnerney P. Methodology for JBI scoping reviews. In: The Joanna Briggs Institute Reviewers' Manual. The Joanna Briggs Institute, 2015: 1-24.

23 Arksey H, O'Malley L. Scoping studies: towards a methodological framework. Int J Soc Res Methodol 2005;8:19-32.

24 Tricco AC, Lillie E, Zarin W, et al. PRISMA extension for scoping reviews (PRISMA-ScR): checklist and explanation. Ann Intern Med 2018;169:467-73.

25 +Government of Canada Cl of HR. Health Research in Canada and You - ClHR, 2011. Available: https://cihr-irsc.gc.ca/e/43753.html [Accessed 20 Jul 2020].

26 Gostin LO, Levit LA, Nass SJ. Beyond the HIPAA privacy rule: enhancing privacy, improving health through research. National Academies Press, 2009.

27 Hoekstra F, Mrklas KJ, Khan M, et al. A review of reviews on principles, strategies, outcomes and impacts of research partnerships approaches: a first step in synthesising the research partnership literature. Health Res Policy Syst 2020;18:51.

28 Peters M, Godfrey C, Mclnerney P. Chapter 11: Scoping Reviews (2020 version). In: Aromataris E, Munn Z, eds. Joanna Briggs Institute Reviewer's Manual. In: JBI, 2020. https://reviewersmanual. joannabriggs.org/

29 Canadian Agency for Drugs and Technologies in Health. Grey matters: a practical tool for searching health-related grey literature, 2020. Available: https://www.cadth.ca/resources/finding-evidence/ grey-matters

30 Covidence - Better systematic review management. Available: https://www.covidence.org/home [Accessed 13 Dec 2019].
31 Research Excellence Framework. Assessment framework and guidance on submissions, 2011. Available: https://www.ref.ac.uk/ 2014/media/ref/content/pub/assessmentframeworkandguidanceon submissions/GOS\%20including\%20addendum.pdf

32 Definition of a health outcome. Research, 2015. Available: https:// uwaterloo.ca/research/office-research-ethics/research-humanparticipants/pre-submission-and-training/human-researchguidelines-and-policies-alphabetical-list/definition-health-outcome [Accessed 6 Aug 2020].

33 Graham ID, Logan J, Harrison MB, et al. Lost in knowledge translation: time for a map? J Contin Educ Health Prof 2006;26:13-24.

34 IAP2 Canada - Public Participation Spectrum. Available: http:// iap2canada.ca/page-1020549 [Accessed 10 Oct 2017].

35 Albrecht L, Archibald M, Arseneau D, et al. Development of a checklist to assess the quality of reporting of knowledge translation interventions using the Workgroup for intervention development and evaluation research (WIDER) recommendations. Implement Sci 2013;8:52.

36 Dillon EC, Tuzzio L, Madrid S, et al. Measuring the impact of Patient-Engaged research: how a methods workshop identified critical outcomes of research engagement. J Patient Cent Res Rev 2017;4:237-46.

37 Michie S, Atkins L, West R. The Behaviour Change Wheel (Behavior Change Wheel) - A Guide To Designing Interventions. Silverback Publishing, 2014

38 Atkins L, Francis J, Islam R, et al. A guide to using the theoretical domains framework of behaviour change to investigate implementation problems. Implement Sci 2017;12:77.

39 Braun V, Clarke V. Using thematic analysis in psychology. Qual Res Psychol 2006;3:77-101.

40 Ovretveit J, Hempel S, Magnabosco JL, et al. Guidance for researchpractice partnerships (R-PPs) and Collaborative research. $J$ Health Organ Manag 2014;28:115-26.

41 Hoekstra F, Mrklas KJ, Sibley KM, et al. A review protocol on research partnerships: a coordinated multicenter team approach. Syst Rev 2018;7:217.

42 Nguyen T, Graham ID, Mrklas KJ, et al. How does integrated knowledge translation (IKT) compare to other Collaborative research approaches to generating and translating knowledge? learning from experts in the field. Health Res Policy Syst 2020;18:35.

43 barrier noun - Definition, pictures, pronunciation and usage notes | Oxford Advanced Learner's Dictionary at OxfordLearnersDictio naries.com. Available: https://www.oxfordlearnersdictionaries.com/ definition/english/barrier?q=barrier [Accessed 7 May 2020].

44 Facilitator | definition of facilitator by Merriam-Webster. Available: https://www.merriam-webster.com/dictionary/facilitator [Accessed 16 Jul 2020]. 